\title{
Malignant Melanoma Arising De Novo Within A T.A.B.T. Inoculation Site
}

\author{
Major G J Attard \\ FRCS(Ed), RAMC
}

Queen Elizabeth Military Hospital, Woolwich SE186XN

SUMMARY: A case of malignant melanoma arising in a T.A.B.T. inoculation site is reported. Any progressive change within a inoculation scar, or immunisation site, should be thoroughly evaluated and treated appropriately after tissue diagnosis.

\section{Case Report}

A 35-year-old soldier presented with a five year history of a painless lesion on his upper arm. This developed shortly after and at the site of immunisation with T.A.B.T. (Typhoid - paratyphoid A\&B and Tetanus Vaccine). The area had remained erythematous and occasionally discharged a clear fluid. Over the preceeding months it had enlarged and bled when traumatised. On examination, there was a slightly raised erythematous area $1 \frac{1}{2} \mathrm{cms}$ diameter with an irregular outline. There was no lymphadenopathy.

The patient was first seen by a dermatologist. The clinical diagnosis was uncertain, but a patch of chronic discoid lupus was considered the most likely. An incision biopsy reported a malignant melanoma.

The patient was referred to the Oncology Unit, and a wide excision carried out. Histology revealed a superficial spreading malignant melanoma with invasion to Clarke's level 2. (tumour involving papillary dermis with a depth of invasion less than $0.75 \mathrm{~mm}$ ).

The patient remains disease free at five years.

\section{Discussion}

A variety of malignant tumours have been described arising in vaccination scar sites. According to Kulwin ${ }^{1}$ a total of 54 cases of malignant change had been reported arising in smallpox vaccination scars. Of these, 31 were basal cell carcinomas, nine were squamous cell carcinomas, and 13 were malignant melanomas. Malignant melanomas accounted for a surprising $24 \%$ of the cases.

Lokich $^{2}$ described a case of malignant melanoma arising within a BCG scarification site.

While it is interesting to speculate on these clinical oddities, it is worth remembering that almost a quarter of the reported cases have been malignant melanomas. Complete excision of a suspicious lesion in an inoculation scar or site with a small clear margin for diagnostic purposes is indicated. Incision biopsy may not only give unrepresentative areas of the lesion to the histologist, but may also carry a risk of surgically induced metastases.

\section{Acknowledgements}

I am grateful to Colonel N. Peters L/RAMC for permission to report this case.

\section{REFERENCES}

1 KULwIN M H. Basal cell epithelioma in smallpox vaccination scars-fifty years later. Ill Med J 1975.

2 LOKICH J J. Malignant melanoma arising de novo within a BCG scarification site. Lancet (Letter) 1975;1:331-332. 